\title{
Prácticas, saberes y apropiación del medio audiovisual en los colectivos de comunicación comunitaria de la ciudad de Medellín
}

Juan Gonzalo Lopera Rueda*

DOI: https://doi.org/10.33571/revistaluciernaga.v11n22a2

\section{Resumen}

En el artículo se presenta la reflexión producto de investigación de las prácticas, saberes y apropiación que del audiovisual agencian los colectivos de comunicación comunitaria: ComúnAudiovisual, Pasolini en Medellín y Mancha Negra, todos ellos con incidencia en barrios periféricos de la ciudad de Medellín.

Los audiovisuales de estos colectivos son el resultado de procesos investigativos y metodológicos realizados en amalgama con la comunidad. Esos Audiovisuales Comunitarios (AC); Documentales Sociales Participativos (DSP); Documentales Etnográficos (DE) contienen la memoria y vivencias de las comunidades. Inciden en la identidad y construcción del tejido social comunitario. Comunicación otra, aquella del buen vivir, el cambio social que impulsa a expresarse y al diálogo de saberes.

Palabras clave: Medellín; comunicación; colectivos; medios; alternativos; audiovisual; videos; cambio social.

Recibido. Mayo 07, $2019 \quad$ Aceptado. Mayo 23,2019 


\section{Practices, knowledge and appropriation of the audiovisual media in the community communication collectives in Medellin city}

Juan Gonzalo Lopera Rueda*

DOI: https://doi.org/10.33571/revistaluciernaga.v11n22a2

\section{Abstract}

The article presents the reflection resulting from research into the practices, knowledge and appropriation of audiovisuals by community communication collectives: ComúnAudiovisual, Pasolini in Medellín and Mancha Negra, all of them with an incidence in suburban areas in the city of Medellín. The audiovisuals of these collectives are the result of research and methodological processes carried out in alliance with the community.

These Community Audiovisuals (CA); Participatory Social Documentaries (DSP); Ethnographic Documentaries (DE) reflect the memory and experiences of the communities. They have an impact on the identity and construction of the community's social fabric. Communication, the one of good life, the social change that encourages expression and the dialogue of knowledge.

Key words: Medellín; communication; collective; alternative means; audio-visual; videos; social exchange.

Received. May 7, $2019 \quad$ Accepted. May 23,2019

Traducir el resumen y la información del autor 


\section{Práticas, conhecimentos e apropriação dos meios audiovisuais nos colectivos comunitários de comunicação da cidade de Medellín}

Juan Gonzalo Lopera Rueda*

DOI: https://doi.org/10.33571/revistaluciernaga.v11n22a2

\section{Resumo}

O artigo apresenta a reflexão resultante da investigação sobre as práticas, conhecimentos e apropriação do audiovisual pelos colectivos comunitários de comunicação: ComúnAudiovisual, Pasolini em Medellín e Mancha Negra, todos eles com impacto nos bairros periféricos da cidade de Medellín.

O audiovisuais destes colectivos são o resultado de de processos investigativos e metodológicos realizados em fusão com a comunidade. Estes Audiovisuais Comunitários (AC); Documentários Sociais Participativos (DSP); Documentários Etnográficos (DE) contêm a memória e as experiências das comunidades. Elas influenciam a identidade e a construção do tecido social comunitário. A comunicação é outra, a da boa vida, a mudança social que incentiva a expressão e o diálogo de conhecimentos.

Palavras chave: Medellín; comunicação; colectivos; media; alternativa; audiovisual; vídeos; mudança social.

Recebido. Maio 07, $2019 \quad$ Aceitado. Maio 23, 2019 


\section{Introducción}

En la ciudad de Medellín se cuenta con una amplia trayectoria en lo referido a medios de comunicación comunitaria, se observa en este lugar una gama de experiencias y esfuerzos de colectivos de comunicación y centros de producción audiovisual comunitaria de disimiles perspectivas filosóficas, sociopolíticas y culturales. Hay una heterogeneidad de medios comunitarios que pueden ser populares, alternativos o ciudadanos, dependiendo de la base social a la que impactan y las necesidades a las que apuntan comunicacionalmente. Aspectos que fundamentan sus agendas y gestión de sus procesos psicosociales, sociopolíticos, culturales y estéticos.

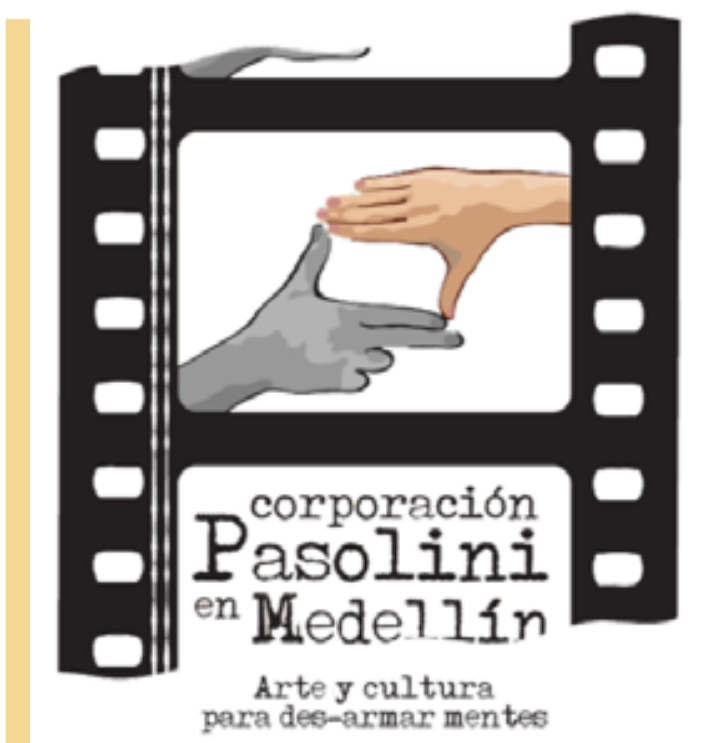

El colectivo ComúnAudiovisual está ubicado en la Comuna 8, Pasolini en Medellín tiene incidencia en las comunas 13, 1, 8, 16, 10, de la ciudad y Mancha Negra realiza sus acciones en la comuna 1. Estas experiencias comunitarias al igual que otras similares, cuentan con poca visibilidad en el contexto general de la ciudad. Es usual que cada colectivo con sus propios recursos y esfuerzos lleve a cabo sus prácticas y labores comunicativas.
Este artículo se enfoca en esa "otra comunicación posible" ( Sierra \& Gravante, 2012, pág. 130), aquella que está al lado de las comunidades y se gesta a partir de sus necesidades de expresión y reconocimiento social, político y cultural. En tal sentido, se parte del objetivo de caracterizar, visibilizar las prácticas, saberes y apropiaciones que ponen en circulación los colectivos audiovisuales comunitarios. En otras palabras, pretendemos evidenciar a estos colectivos que en unión con comunidades activas generan voces propias, ciudadanías críticas, movilizan saberes y hasta presionan la agenda pública.

En tal sentido el foco se ubicó en esa comunicación que provoca rupturas y provee nuevas lecturas de lo que somos, como lo expresa Rincón (2009), esa comunicación en la que es más importante el proceso comunicacional que el producto Gumucio (2011).

Los lentes teóricos que orientaron este estudio se cimentaron en las líneas de la comunicación para el cambio social, comunitaria y ciudadana a partir de las referencias de Gumucio, 2011; Sierra y Gravante, 2012; Cadavid, 2011; y Rodríguez, 2012. También se incluyó la comunicación popular con su énfasis en la mediación y la pregunta por los vínculos entre las agendas de país y la formación del comunicador (Martín Barbero, 1987, 2009) así como las indagaciones sobre los colectivos de comunicación y sus prácticas (Acosta y Garcés, 2013). Convergen también temas de la cultura popular, el contra discurso (Rincón, 2009, 2011) y la línea de la enunciación y la pregunta por el funcionamiento de la información como discurso (Charaudeau, 2003). Se consideran pertinentes las indagaciones por el conocimiento, los saberes situados y los contenidos, abordados por Agüero Servin, 2011; Carlo Piazzini, 2014; Polleri, 2014 y Paredes, 2011. 


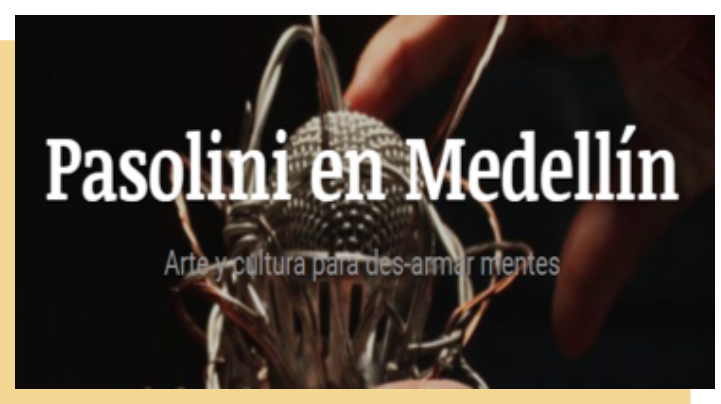

Metodológicamente se optó por la ruta del diálogo de saberes, la investigación cualitativa de tipo etnográfico hermenéutico además de la observación participativa. Las herramientas y técnicas utilizadas en el estudio fueron los diarios de campo y guías de observación, el análisis, documental y clasificación del material producido por los colectivos tales como los videos, registros fotográficos, talleres de sondeo, fichas de descripción y entrevistas.

\section{Prácticas más allá del medio en los colectivos de comunicación comunitaria.}

Los colectivos audiovisuales comunitarios entre ellos los populares, alternativos y ciudadanos, tienen distintas posiciones políticas, culturales, sociales, académicas y de acción en los contextos donde inciden. Todos comparte el propósito de congregar las comunidades en torno a la comunicación para el cambio social y llevar a cabo prácticas y metodologías propias para que el proceso comunicativo y mediático a través de los Audiovisuales Comunitarios (AC), Documentales Sociales Participativos (DSP), Documentales Etnográficos (DE), y Pedagogías Populares en Comunicación (PPC), se empodere en la ciudad de Medellín para la movilización sociopolítica y cultural.

Las realización y producción audiovisual de cada colectivo se caracteriza por su especificidad. ComúnAudiovisual y Mancha Negra abordan el audiovisual comunitario (AC) y los documentales sociales participativos (DSP). Pasolini en Medellín los documentales etnográficos (DE). En ellos se evidencia la participación de las comunidades. Para estos colectivos las prácticas audiovisuales tienen sentido al ser realizadas conjuntamente entre el colectivo y la comunidad.

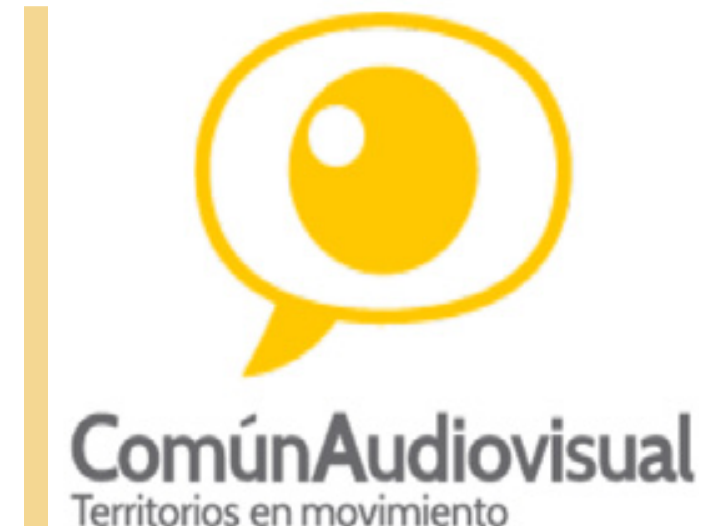

Cada colectivo define su proceder comunicacional. Sostienen un punto de convergencia: el diálogo de saberes y la reivindicación de la comunicación como práctica para la apropiación y la emancipación. En estos colectivos la función pedagógica de intercambio de conocimiento y gestión de los saberes cumplen una labor primordial en el restablecimiento del tejido social de las comunidades. Se presenta una constante retroalimentación del saber en todos los ámbitos de las prácticas comunicativas, para establecer una comunicación audiovisual que vaya más allá del medio y se instaura como el camino para la autonomía social que obra más allá del artilugio mediático hegemónico o institucional; buscan el acercamiento a la realidad de las comunidades al expandir el espectro poderoso que los medios desde su función social poseen.

Estos colectivos son conscientes que tiene más validez el proceso comunicativo que el mismo dispositivo o instrumento audiovisual. Validan que la comunicación en principio es intercambio y diálogo construido en común que sirve para refirmar las voces acalladas que defienden el pensamiento comunitario, ciudadano, político. Máxime en una ciudad altamente inequitativa como Medellín donde la concentración de medios promulga un 
valle de marca registrada con glosarios abstraídos de la mercadotecnia y acomodados a un discurso que trata de incrustarse en las miradas populares.

Los audiovisuales producidos por esos colectivos, de forma contraria al establecimiento, hacen veraz la alocución contestataria y contra hegemónica de las comunidades. Esa que surge en el seno del pueblo organizado y gestado en muchas ocasiones por el desamparo y deficiente ordenamiento gubernamental, pero acrecentadoenla unidad comunitaria.Voces muchas veces contenidas que despiertan como eco estridente en los medios creados entre colectivo - comunidad - territorio; no con el fin de reconocerse individualmente o como distinción estratificada al tener mayores conocimientos, sino en el proceso concertado, mancomunado, en convite, en minga, entre muchos. Ese conocimiento que recoge el saber situado y lo expande y que deja un documento valioso de memoria comunitaria.

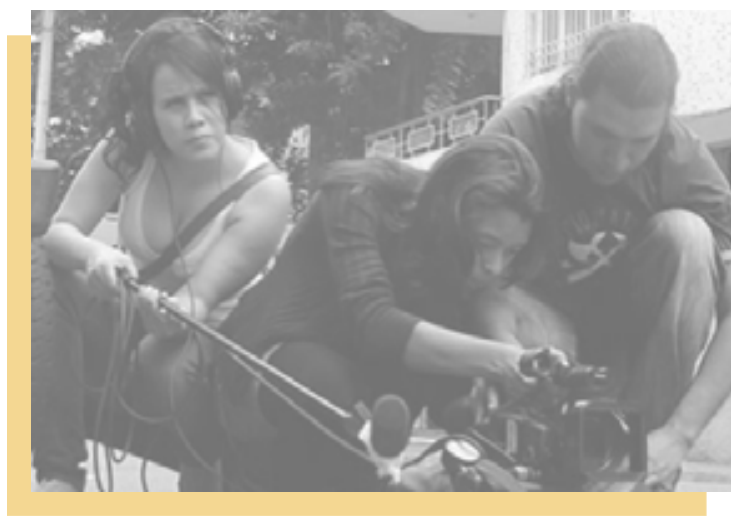

En estos colectivos de comunicación audiovisual comunitaria se observa gestión, planeación estratégica, estructura organizativa, así como pensamiento social, filosófico, político y cultural.

Las formas organizativas de estos colectivos no comprometen su carácter de independencia y acción, estos adaptan creativamente estructuras tradicionales de realización y organización, priorizan necesidades y establecen un orden propio en las dinámicas temporales donde las labores trasiegan, bajo estados de tiempo y espacialidad no convencionales. En los procesos organizativo estratégicos se observa departamentalización, en los roles de producción y acciones académico pedagógicas, se asumen responsabilidades todo ello sin perder su distintivo comunitario. Además, se preserva el carácter político desde el que han fijado su acción social; son consecuentes con sus finalidades, todo esto con la anuencia de las comunidades y la construcción de medios con el lenguaje de las comunidades sus gramáticas y fonéticas.

Se resalta el bagaje de saberes y conocimientos que se concentra en estos colectivos, lo cual es consecuente con sus iniciativas sociales y fruto de la experiencia y recorrido por varios años en la comunicación para el cambio social, lo que les permite el diálogo con las comunidades y su reconocimiento.

Estos colectivos generan dinámicas sociales en los territorios, están al lado de la comunidad, abren espacios para la cultura, la información, la comunicación y el saber. Forjan otras formas de observar el mundo, velar por los derechos y esclarecer deberes. Labor emancipadora que abre y expande miradas e identidades. En sus audiovisuales las comunidades se reconocen como habitantes de una ciudad que los debe escuchar, un país que los debe ver, un planeta que los debe sentir.

En estos colectivos, las tensiones creativas en la realización de los productos audiovisuales, se reconoce como diferencia que se potencia con los puntos de vista de los involucrados; lo individual se colectiviza para acrecentar el saber comunicacional; siempre el relato será más importante que el dato, esto significa que, la voz de la comunidad, el habitante, el ciudadano, el comunicador comunitario, popular y etnográfico es privilegiada. Desde esta perspectiva se lleva a cabo la producción de los AC, DSP y DE [1].

Las prácticas comunicativas audiovisuales inciden en la identidad y construcción del tejido social comunitario. Comunicación 
otra, aquella del buen vivir, el cambio social que incita a expresarse. De estas producciones audiovisuales surgen iniciativas que retoman las comunidades para dinamizar acciones y agendar temáticas. En tal sentido este tipo de producción aporta a la promulgación de la opinión pública comunitaria.

También los AC, DSP, DE [2] son espacios para la deliberación social tanto para los colectivos como para los agentes sociales, organizaciones, y comunicadores populares que vislumbran en estos un lugar para confrontar y desvelar saberes; difundir, rescatar y promover las memorias comunitarias; esgrimir, demandar y construir acciones movilizadoras.

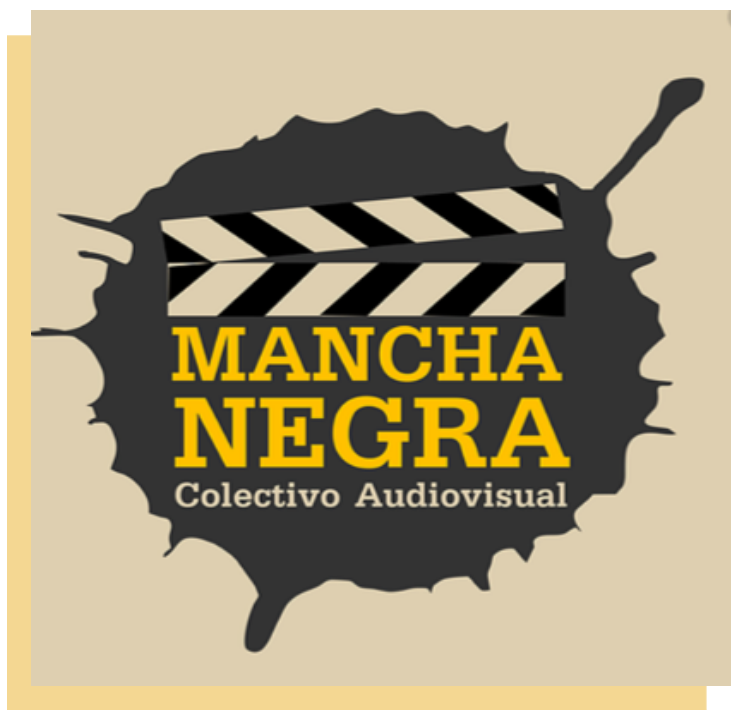

Los colectivos de comunicación audiovisual comunitaria llámense populares, alternativos o ciudadanos han forjado una historia y lucha, que en muchos casos ha sido deslegitimada en sus planteamientos sociopolíticos, situación que devalúa la labor que cumplen en las comunidades donde su tarea fundamental se concentra en dar visibilidad a las problemáticas sociales, expresar el derecho legítimo a la protesta y enunciar agendas críticas. Fortalecen las ciudadanías, vinculándolas políticamente en las decisiones que les afectan ( Niño \& Jiménez, 2015).

Estos colectivos demandan ante la opinión pública su derecho a ser tenidos en cuenta, inclusión en las políticas públicas de comunicación, acceso a pantallas y reconocimiento de su labor social en las comunidades, así como su trabajo en pro de la civilidad, reconciliación, inclusión, respeto e igualdad social.

\section{Movilizar los saberes}

En cada uno de los colectivos de comunicación audiovisual se observa una constante retroalimentación del saber en todos los ámbitos de las prácticas comunicativas. La Investigación - Acción - Participación y la Etnografía Audiovisual se implementan como metodologías de trabajo. La autoevaluación es continua; realizan revisionesy ajustes de sus acciones; se motiva la experimentación y nuevas formas en los procesos comunicativos. La gestión, producción y realización audiovisual de sus documentales [3] cuenta con procedimientos explícitos.

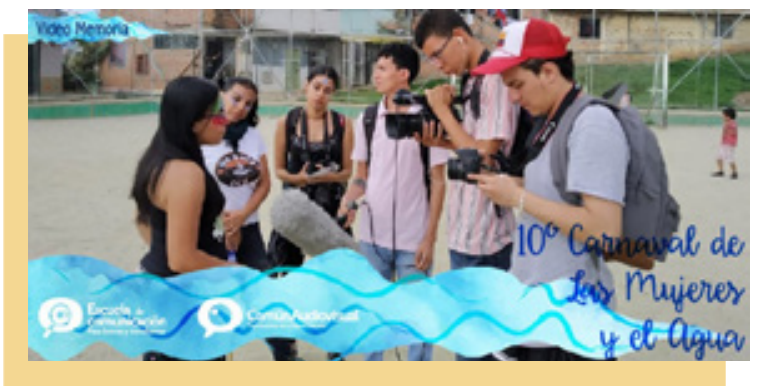

Tomado de: http://www.ciudadcomuna.org/ ciudadcomuna/16-medios/comunaudiovisual.

html?layout=

El colectivo ComúnAudiovisual se acerca a la base social por medios de los Comité de Contenidos; ejercicio de inclusión social que simula una sala, reunión de redacción o producción pero que dista sustancialmente en sus acepciones de las formas tradicionales; este comité obra de manera autónoma y desde el sentir comunitario. En este espacio se argumenta, se consideran y definen los audiovisuales a partir de temáticas propuestas por las comunidades. Esta práctica implica el diálogo de saberes que parte de un tema de importancia comunitaria, no hay censura para su abordaje; en el debate participan diferentes grupos generacionales y representantes de los territorios. El diálogo es horizontal, plural. Este espacio 
sirve además como catarsis, enseñanza y aprendizaje social.

Mancha Negra por su parte, aplica Pedagogía Popular en Comunicación y Campañas de Comunicación Popular, tienen Semilleros de Comunicación con los que pretenden incrementar el reconocimiento de la sabiduría autóctona. Para ellos los medios más que un fin son parte del proceso que lleva al reconocimiento sociopolítico y cultural. La contracultura y la resistencia es el eco que retumba en las manifestaciones comunicativas promovidas por este colectivo; la participación comunitaria es el eje primordial para la reivindicación social y visibilidad en las agendas públicas, esenciales para la movilización y el cambio social.

Pasolini en Medellín recurre a la Etnografía Dialógica. En la Cocina de Guiones, comparten y generan lazos de cercanía, amistad y empatía con las comunidades; esto les permite acercarse al relato, encontrar narrativas desde quien o quienes las viven y sienten. Es así como a partir de la cocción de un plato de comida y la aplicación de métodos de la Investigación Acción Participativa se cuecen las historias.

En esta actividad se fusiona la gastronomía, la memoria, la identidad, la cartografía, fotografía e historias de vida; todo ello en intercambio dialógico sin marcas ni podios. De esta manera surgen las historias que dan origen a las categorías temáticas de la producción audiovisual. La construcción narrativa tiene sentido por las acciones que expresan las comunidades y que se desarrollan en los diferentes audiovisuales.

\section{Apropiados: muchas voces exclamadas}

La apropiación en la participación de los distintos estadios comunicativos audiovisuales que proyectan los colectivos comunitarios referidos en este estudio establece en primera medida una base social asentada en los territorios por lo general de ladera y borde oriental de la ciudad de Medellín.

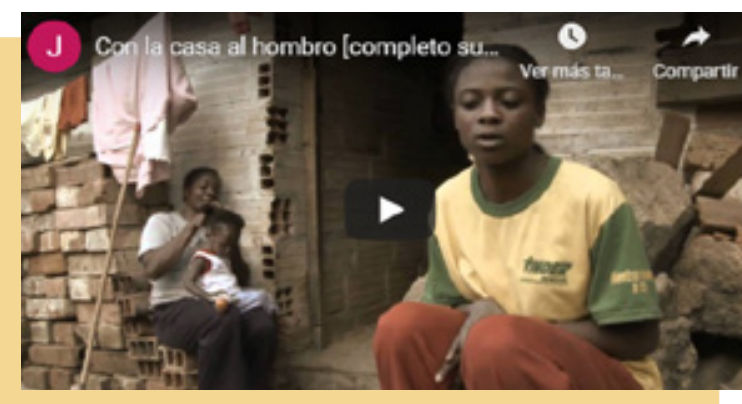

Disponible en: https://pasolinienmedellin.wordpress. com/about/

Las zonas en las que hacen presencia los colectivos audiovisuales en Medellín son: Zona 1 Nororiental. Comunas: 1 popular 2 Santa Cruz - 3 Manrique; Zona 3 Centro Oriental. Comunas: 8 Villa Hermosa - 9 Buenos Aires; Zona Centro Occidental. Comuna: 13 San Javier. Estos lugares convergen organizaciones sociales, gestores culturales, líderes comunitarios y mesas de trabajo, como: mujeres, niñez, juventud, LGBTI, desplazados, desconectados de servicios públicos, mesas de paz, mejoramiento urbano, entre otras; por lo general las comunidades pertenecen a estratos que están entre el 1 y 3.

El papel de la mediación cultural es determinante en la apropiación del medio audiovisual. Todo aquel acervo de identidad y configuración que se da en los territorios cobra mayor relevancia al ser difundido y puesto en público en los medios comunitarios, populares y etnográficos. Emergen los saberes propios y situados, aquellos que narran el sentir, la vivencia y cultura popular.

La apropiación social del medio audiovisual y su empoderamiento tanto en colectivos como en comunidades permiten reconocer las identidades comunitarias, pedagogías populares, políticas, movilizaciones, arraigos, saberes situados de conocimiento y creencia, territorios, gramáticas, consensos, amistad, narraciones, relatos endógenos y mediaciones culturales; es decir, todo un compendio de expresiones con función y sentido sociopolítico. 
Colectivos como ComúnAudiovisual, Mancha Negra y Pasolini en Medellín han abierto un camino expedito de experiencias y saberes construidos alrededor del diálogo y la comunicación como proceso y práctica de lo social amparados en los conocimientos populares, académicos, experienciales y vivenciales en su trasegar investigativo y socialización metodológica en sus áreas del saber endémico con las comunidades.

En los colectivos de comunicación audiovisual, objeto de estudio, el foco de atención está en las comunidades y los territorios. En la emergencia de los saberes su accionar se erige en pro de visibilizar problemáticas y necesidades de sus comunidades para ser expuestas en las agendas públicas. Esto a partir de la implementación de planes de comunicación y gestión de medios; acciones pedagógicas, populares e integración socio-espacial.

En cada uno de los colectivos, se observa que el proceso delacomunicación sefundamenta en la investigación y el aprendizaje. El saber situado, eventualidades territoriales, integración interorganizacional y decisiones de orden habitual en las colectividades es compartido con las comunidades. Con este conocimiento se producen medios y prácticas comunicacionales.

Es vital que los colectivos de comunicación trabajen mancomunadamente con otros pares de su área, este tipo de encuentros se han realizado ocasionalmente y se diluyen al finalizar el proceso que los junto. Se hace imperante buscar unidad con otros agentes comunitarios comunicativos de la ciudad de Medellín. Ésto es, tejer redes que incluyan todas las periferias y bordes citadinos e ir integrándose más allá de los contextos locales.

La independencia audiovisual que rodea los colectivos de comunicación comunitaria, popular y etnográfica es propicia para orientar posiciones políticas con respecto a la situación social que acaece en las comunidades. Se debe tratar de integrar el bien común y buscar una representación ciudadana que les posibilite acceder a recursos gubernamentales para generar proyectos articulados. Construir comunidad integrada.

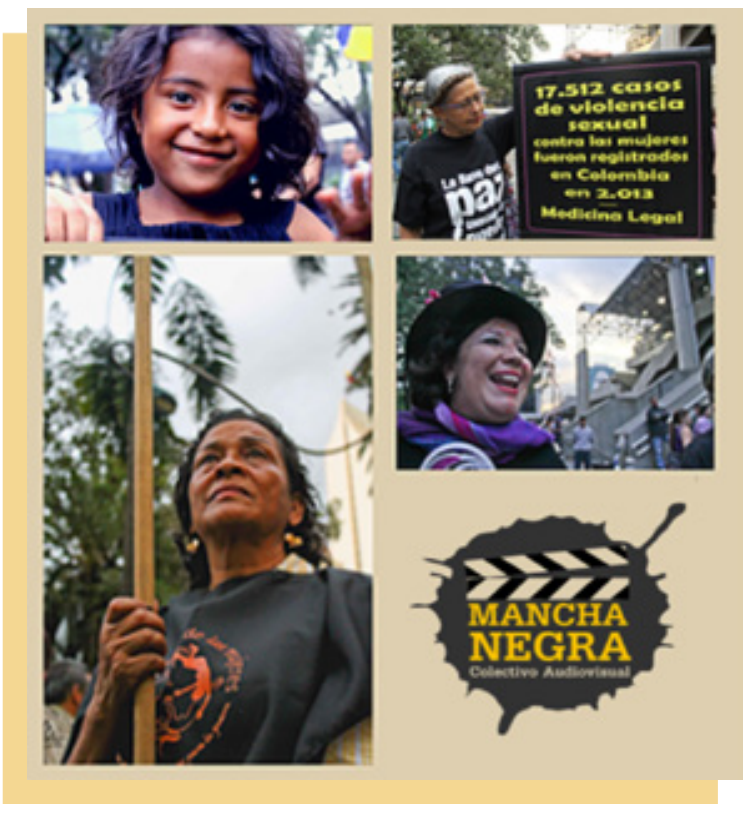

Tomado de : https://bit.ly/2WBIE0Q

Es importante fortalecer los puentes de diálogo con la academia. Las relaciones con la universidad deben permanecer presentes, en tanto es un aliado para la construcción de los procesos y prácticas. Los colectivos constantemente deben ir a la búsqueda de nuevos conocimientos que repercutan en saberes para adaptarlos y multiplicarlos en la gestión organizacional y estratégica a partir de metodologías para la investigación y creación particular.

En los colectivos se deben fomentar formas de participación social y empoderar los medios de comunicación audiovisual para la creación de narrativas originales y situadas alejadas de la institucionalidad de la media y más cercana a las identidades del territorio.

Debe ser primordial en los colectivos recobrar las memorias comunitarias y populares. Apropiar y proponer el agenciamiento de temáticas que impulsen la reflexión en las identidades, expresiones sociales latentes y los capitales intangibles. De igual forma la gestión, valoración de los saberes y creencias propias. 
Entre mayoraperturay ángulos de distinción posean los colectivos más fuerte será su capacidad de relacionar los elementos de las prácticas comunicativas para los audiovisuales y mantener la expansión de criterios en diferentes formas de percibir la comunicación como fundamento en los procesos sociales. Al poseer el medio comunitario, popular y etnográfico esa característica de pluralidad y convergencia con las demandas y expresiones de las comunidades la relación asimétrica y horizontal se sostiene.

En este contexto, el conocimiento en gestión socio cultural, comunicación comunitaria y movimientos sociales son de gran competencia. La motivación comunitaria es fundamental, ésta es el cimiento que sostiene los procesos comunicativos, permite emerger el saber y nutrir el diálogo, afianzar los lazos y fortalecer las vivencias.

Formar en comunicación audiovisual de manera crítica es fundamental, desde esta perspectiva se da sentido a la apropiación mediática con sentido social, así como a la integración de la academia con las comunidades. Aportan a esta formación las metodologías propias y abstraída de las etnografías dialógicas y la investigación participativa.

Los procesos de comunicación audiovisual comunitaria y afines con sus prácticas, saberes y resultados sirven como terreno de confrontación ante lo mediático instituido, también como ventana abierta de las realidades vividas en los territorios y ampliadas bajo la mirada de las comunidades que al unísono con los colectivos expresan la situación vivida en los contextos territoriales donde la desigualdad, carencias, demandas, acciones, procesos, y en sí la movilización social es expresadas sin sesgo o censura mediática.

La apropiación comunicativa audiovisual en los colectivos en relación con las comunidades les empodera elementos abstraídos de la Pedagogía Popular en Comunicación, la Investigación Acción Participación y la Etnografía Dialógico Audiovisual, conocimientos que se relacionan con los saberes situados y proporcionan mayor gestión del medio para orientar los relatos en las comunidades.

La apropiación de la comunicación y realización audiovisual comunitaria sea popular, alternativa, ciudadana u otras a partir de los AC, DSP, DE [4] gestionados entre colectivos y comunidad, promueve agendas de opinión pública.

El empoderamiento comunicativo audiovisual en proceso de creación conjunta entre colectivos y comunidades trasciende la visión instrumentalista de los medios.

Los colectivos de comunicación, en el entorno colombiano, deben incidir en los planes de gobierno a nivel local, departamental y nacional. En tal sentido les corresponde ser partícipes de las políticas públicas comunicativas máxime en el contexto del Acuerdo de Paz y el Posconflicto.

Otros estudio de estos procesos y prácticas de comunicación en los colectivos de orden comunitario están por abordarse, entre estos: auscultar la relación territorialidad, comunidad, juventud y comunicación comunitaria; acciones sociopolíticas y culturales en relación a la comunicación y el discurso audiovisual comunitario; la contracultura audiovisual comunitaria; las metodologías comunitarias para la creación del audiovisual comunitario; el post acuerdo de paz colombiano y el audiovisual en las comunidades como herramienta de socialización; además de otras formas comunicativas de orden social que están a la orden del día porque de la comunicación para el cambio social y las prácticas audiovisuales comunitarias aún queda mucho por desvelar, por aprender y desaprender en el universo comunicacional audiovisual. 


\section{Referencias}

Acosta , G. L. (2013). Gestión De Medios Para La Movilización y El Cambio Social : Un análisis discursivo de las prácticas de comunicación en colectivos de Medellín. Neila et alter (Comp.). Estudios del Discurso en América Latina. Homenaje a Anamaría Harvey. Asociación Latinoamericana de Estudios del Discurso, ALED., Bogotá.

Acosta, G. L., \& Garcés, Á. (2013). Colectivos de comunicación y apropiación de medios. Medellín: Sello Editorial Universidad de Medellín.

Acosta, G. L., \& Garcés, Á. (2013). Colectivos de comunicación y apropiación de medios. Medellín: Sello Editorial Universidad de Medellín.

Álvarez, P. (2004). Colombia. En A. Gumucio, \& M. P. Hoyos (Ed.), El cine comunitario en América Latina y el Caribe. Bogota, Colombia: Centro de Competencia en Comunicación para América Latina, C3.

Álvarez, S. , \& Paz , S. (2013). /www. aacademica.com. Recuperado el 13 de Agosto de 2014, de /www.aacademica. com: www.aacademica.com/000$076 / 20$

Aguilera , C., \& Polanco, G. (Diciembre de 2011). Videocomunidad - Prácticas, procesos y sentidos audiovisuales. Recuperado el 13 de Agosto de 2014, de Videocomunidad - Prácticas, procesos y sentidos audiovisuales: http://videocomunidad.univalle.edu.co/ libros.html

Bustos, G. (2006). Audiovisuales de combate. Acerca del videoactivismo cintemporáneo. Buenos Aires, Argentina: Editorial La Crujía Ediciones - CCEBA.

Cadavid, A. (2011). La Comunicación para el Desarrollo en Colombia, los Últimos 20 años. En J. Pereira, \& A. Cadavid, comunicación desarrollo y cambio social:Interrelaciones entre comunicación, movimientos ciudadanos y medios. (págs. 57-78). Bogotá: Pontificia Universidad Javeriana.

Carvajal, K. E. (29 de febrero de 2016). Mancha Negra. (L. R. Gonzalo, Entrevistador)
Chacón, J. (2017). Características de los medios comunitarios y alternativos de Bogotá- Colombia. Luciérnaga, 8(15), 63-83. doi:https://doi.org/10.33571/ revistaluciernaga.v8n15a5

Charaudeau, P. (2003). Una definición discursiva de la información. En P. Charaudeau, El Discurso de la Información. La construcción del. Barcelona: Gedisa.

ComúnAudivisual. (15 de Enero de 2016). ComúnAudiovisual. (L. R. Gonzalo, Entrevistador)

Cárdenas, N., \& Gómez, E. (2009). El audiovisual como identificador de identidades políticas de la realidad juvenil. Trabajo de grado, Universidad del Norte, División de Humanidades y Ciencias Sociales, Programa de Comunicación Social, Barranquilla.

D’abbraccio Krentzer, G. (2016). La Industria del Cine en Colombia. Entre el optimismo ingenuo y el pesimismo crónico. Luciérnaga, 7(14), 36-45. doi:https://doi.org/10.33571/ revistaluciernaga.v7n14a1

De Agüero Servin, M. d. (Septiembre Diciembre de 2011). Conceptualización de los saberes y el conocimiento. (C. V. Tamez, \& M. Calderón García, Edits.) Desicio(30), 16 - 20.

Durán, O. (julio de 2012). Fes Media Latin america. Recuperado el 18 de Septiembre de 2014, de Fes Media Latin america: http://www.fesmedialatin-america.org/inicio/resultados-debusqueda/?L=0

Gumucio, A. (2011). comunicación para el cambio social: claves de desarrollo participativo. En J. M. Pereira, \& A. Cadavid, comunicación desarrollo y cambio social: Interrelaciones entre comunicación, movimientos ciudadanosy medio. Bogota, Cundiamarca, Colombia: Pontificia Universidad Javeriana.

Franco, C. E. (Septiembre de 2011). Repositorio Institucional Universidad Pontificia Bolivariana. Recuperado el 16 de Agosto de 2014, de Repositorio Institucional Universidad Pontificia Bolivariana: http://repository.upb.edu. co:8080/jspui/handle/123456789/1596 
Garcés Montoya, Á., \& Jiménez García, L. (2015). Campañas de Comunicación para la Incidencia Política. En L. Jiménez García, Metodologías en Diálogo de Saberes (págs. 12-25). Medellín: Cocorota Inc Publicidad.

Garcés Montoya, Á., \& Jiménez García, L. (2015). Documental Social Participativo DSP-. En L. Jiménez García, Metodologías en Diálogo de Saberes (págs. 65-75). Medellín: Cocorota Inc Publicidad.

Garcés Montoya, Á., \& Jiménez García, L. (2015). Escuela de Comunicación Comunitaria. En L. Jiménez García, Metodologías en Diálogo de Saberes (págs. 48-64). Medellín: Cocorota Inc Publicidad.

Garces, A. (2016). Prácticas en colectivos de comunicación: caso Ciudad Comuna y Pasolini en Medellín. En G. L. Acosta, M. C. Pinto, \& C. A. Tapias (Edits.), Diálogo de saberes en comunicación: Colectivos y Academia.Colección Diálogo de Saberes (Vol. 1). Medellín: Sello Editorial Universidad de Medellín.

Garcés, A., \&Acosta, G. (2013).Apropiación y Gestión de Medios en Colectivos de Comunicación en Medellín. Obtenido de www.academia.edu: www.academia.edu

García, M. (2007). Medios Ciudadanos y Comunitarios en América Latina. En O. Rincón, M. P. Saffon, A. Cadavid, A. E. Villegas, N. Ramírez, M. I. Martínez, . . . J. El'Gazi, Ya No es Posible el Silencio (pág. 262). Bogotá, Cundinamarca, Colombia: Centro de Competencia en Comunicación para América Latina - Friedrich Ebert Stiftung.

Granada, M. (29 de Febrero de 2016). Mancha Negra. (L. R. Gonzalo, Entrevistador)

Greenwood, T., \& Dujardin, M. (15 de enero de 2016). Común Audiovisual. (L. R. Gonzalo, Entrevistador)

Guber, R. (2001). La observación participante. En R. Guber, La etnografía, método, campo y reflexividad (págs. 5574). Bogotá: Grupo Editorial, Norma.

Gumucio, A. (9 de Marzo de 2014). El cine comunitario en américa latina. Bogota: Centro de Competencia en Comunicación para América Latina, C3 FES. Obtenido de Friedrich-EbertStiftung Latinoamerica: http://www. fesmedia-latin-america.org/inicio/ acerca-de-fes/
Hernández , T., \& Moreno, F. (2011). La comunicación comunitaria:vigencia y olvido. En C. Krohling, T. Tufte, \& J. Vega, Trazos de una otra comunicación en América Latina : prácticas comunitarias, teorías y demandas sociales. Barranquilla, Atlantico, Colombia: Editorial Universidad del Norte.

Jiménez García, L. (2015). El Documental Social Participativo - DSP-. En L. Jiménez García, La Comunicación Haciendo Escuela (págs. 46-48). Medellín: Cocotora Inc.

Jiménez, L. (2015). La Comunicación Haciendo Escuela - Diálogo de Saberes Sobre Comunicación Comunitaria en Medellín. Medellín: Fundación Confiar, Corporación Con-Vivamos y Corporación Ciudad Comuna.

Jiménez, L. (2015). Luces,Cámara,Apropiación. Medellín: Ciudad Comuna - Corporación para la Comunicación.

Jiménez, L. (15 de enero de 2016). ComúnAudiovisual. (L. R. Gonzalo, Entrevistador) Medellín, Colombia.

Krohling, C., Tufte, T., \& Vega, J. (2011). Trazos de otra comunicación en América Latina - Prácticas comunitarias, teorías y demandas sociales. Barranquilla, Atlantico, Colombia: Editorial Universidad del Norte. Recuperado el 10 de Septiembre de 2014, de Repositorio Digital - Universidad del Norte: http://manglar.uninorte.edu.co/ handle/10584/1192

López Cerquera, N. (2018). CINE CON MIRADA LOCAL. Práctica política que recupera y resiste. LUCIÉRNAGA, 9(18). doi: https://doi.org/10.33571/ revistaluciernaga.v9n18a1

Mancha Negra. (19 de febrero de 2016). Entrevista a Mancha Negra. (J. G. Lopera Rueda, Entrevistador)

Mancha Negra. (29 de febrero de 2016). Entrevista a Mancha Negra. (L. R. Gonzalo, Entrevistador)

Martín Barbero, J.(2009). www.fesmedialatin-america.org. Recuperado el 13 de Agosto de 2014, de www.fesmedialatin-america.org: www.fesmedia-latinamerica.org/inicio/publicaciones/ entre-saberes-desechables-y-saberesindispensables/ 
Martín-Barbero, J. (1987). De los medios a las mediaciones,Comunicación, cultura y hegemonía. Barcelona, España: Editorial Gustavo Gili, S.A.

Martínez, M. (2004). http://prof.usb.ve/ miguelm/. Recuperado el 4 de Junio de 2015, de http://prof.usb.ve/miguelm/: http://prof.usb.ve/miguelm/articulos. html

Moreira, G., \& Marcus, G. (2011). La construcción de la comunicación comunitaria en la región del Sisal: Una red tejida con fibra y resistencia. En K. Cicilia , T. Tufte, \& J. Vega, Trazos de otra comunicación en América Latina - Prácticas comunitarias, teorías y demandas sociales. Barranquilla, Atlantico, Colombia: Editorial Universidad del Norte.

Niño, E., \& Jiménez, L. (2015). Breve contexto social y político de Medellín en. En L. Jiménez, La comunicación Haciendo Escuela: Diálogo de Saberes sobre Comunicacion Comuitaria en Medellín (págs. 11 - 19). Medellín: Fundación Confiar, Corporación ConVivamos y Corporación Ciudad Comuna.

Polanco, G., \&Aguilera, C. (julio de 2011). Videocomunidad - Practicas, Procesos y Sentidos Audiovisuales en el Suroccidente Colombiano. Recuperado el 13 de Agosto de 2014, de Videocomunidad - Practicas,Procesos y Sentidos Audiovisuales en el Suroccidente Colombiano: http:// videocomunidad.univalle.edu.co/libros. html

Polanco, G., \&Aguilera, C. (julio de 2011). Videocomunidad - Prácticas,Procesos y Sentidos Audiovisuales en el Suroccidente Colombiano. Recuperado el 13 de Agosto de 2014, de Videocomunidad - Prácticas,Procesos y Sentidos Audiovisuales en el Suroccidente Colombiano: http:// videocomunidad.univalle.edu.co/libros. html

Polleri , G. (28 de Mayo de 2014). http:// es.slideshare.net. Recuperado el 10 de mayo de 2015, de http://es.slideshare. net: http://es.slideshare.net/giosiannap/ contenidos-35210755

Paredes, N. (12 de Noviembre de 2011). http://es.slideshare.net/. Recuperado el 10 de Mayo de 2015, de https://bit. ly/31hOMQh
Pasolini en Medellín. (2 de Febrero de 2016). Pasalini en Medellín. (L. R. Gpnzalo, Entrevistador)

Piazzini , C. E. (2014). "Conocimientos situados y pensamientos fronterizos: una relectura desde la universidad". Revista de estudios sobre espacio y poder, 5(1), 11-33.

Piazzini , C. (2014). "Conocimientos situados y pensamientos fronterizos: una relectura desde la universidad". Geopolítica(s). Revista de estudios sobre espacio y poder, 5(1), 11-33.

Pineda, K. (29 de Febrero de 2016). Mancha Negra. (L. R. Gonzalo, Entrevistador)

Real Academia Española. (13 de Agosto de 2016). Obtenido de Real Academia Española: http://dle.rae.es/?id=LYArrlQ

Restrepo, C. (15 de Enero de 2016). ComúnAudiovisual. (L. R. Gonzalo, Entrevistador)

Reguillo, R. (2009). www.fesmedialatin-america.org. Obtenido de www. fesmedia-latin-america.org: www.fesmedia-latin-america.org/inicio/ publicaciones/entre-saberes-desechables-ysaberes-indispensables/

Revista Luciérnaga. (2019). Decreto 1720003875 (2019). Política Pública de Medios y Procesos de Comunicación, Alternativos, Independientes, Comunitarios y Ciudadanos . 11(21), 4. Recuperado de https://revistas.elpoli.edu. co/index.php/luc/article/view/Editorial

Rincón, O. (2007). La Fórmula de la Comunicación Ciudadana: + Ciudadanía + Narración + Activismo. En O. Rincón, M. P. Saffon, A. Cadavid, A. E. Villegas, N. Ramírez, M.I. Martínez, ... J. El'Gazi, Ya No es Posible el Silencio (pág. 262). Bogotá, Cundinamarca, Colombia: Centro de Competencia en Comunicación para América Latina - Friedrich Ebert Stiftung.

Rincón, O. (2009). www.fesmedia-latinamerica.org. Recuperado el 13 de Agosto de 2014, de www.fesmedialatin-america.org:

www.fesmedia-latin-america.org/inicio/ publicaciones/entre-saberes-desechables-ysaberes-indispensables/

Rincón, O. (2011). Estos medios: estéticas activistas y narrativas de uno. En J. M. Pereira, \& A. Cadavid, comunicación desarrolloy cambio social: 
Interrelaciones entre comunicación, movimientos ciuddanos y medios. Bogotá, Cundinamarca, Colombia: Pontificia Universidad Javeriana.

Rodríguez, C. (2011). Trayectoria de un recorrido:comunicación y cambio social en América Latina. En J. M. Pereira , \& A. Cadavid, comunicación desarrollo y cambio social: Interrelaciones entre comunicación, movimientos ciudadanos y medio. Bogotá, Cundinamarca, Colombia: Pontificia Universidad Javeriana .

Rojas Hernández, E. (2015). Cine De Arte y Ensayo En Colombia: Los viajes del viento (2009), El vuelco del cangrejo (2010), La sirga (2012), Porfirio (2012) y La Playa D.C. (2012). LUCIÉRNAGA, 7(14), 1-21. Recuperado de https:// revistas.elpoli.edu.co/index.php/luc/ article/view/815

Rorty, R. (1995). De la Epistemología a la Hermenéutica. En R. Rorty, La filosofía y el espejo de la naturaleza (págs. 287293). Cátedra: Madrid.

Sierra, F., \& Gravante, T. (2012). Apropiación tecnológica y mediación. Líneas y fracturas para pensar otra comunicación posible. En J. Encina, \& M. Á. Ávila, Autogestión de la vida cotidiana.

Sevilla: UNILCO/Editorial Atrapasueños. Recuperado el 15 de Agosto de 2014, de ilusionismosocial.org: ilusionismosocial. org $/ \mathrm{mod} / \mathrm{resource} / \mathrm{view}$.php?id=591

Tapias, C. (2 de Febrero de 2016). Pasolini en Medellín. (L. R. Gonzalo, Entrevistador)

Valle, M., Gómez Sánchez, S., Restrepo, J., Gómez, J., \& Raigosa, W. (2019). Documentales y documentalistas Antioqueños. Sentido y práctica de un oficio que narra una región. LUCIÉRNAGA, 11(21), 5 - 39. doi:https:// doi.org/10.33571/revistaluciernaga. v11n21a1

Vega, J., Pérez, M., Arango, G., \& Pérez, C. (2011). "Pasolini en Medellín": Jóvenes, transferencia de medios y esferas públicas locales. En C. Krohling, T. Thomas, \& J. Vega, Trazos de otra comunicación en América Latina - Prácticas comunitarias, teorías y demandas sociales. Barranquilla, Atlantico, Colombia: Editorial Universidad del Norte.

Villegas, A. E., Ramírez, N., Tunubalá, J., Martínez, M. I., \& Andrade, M. I. (2007). Experiencias (Colectivos de Comunicación Ciudadana en Colombia). En O. Rincón, M. P. Saffon, A. Cadavid, A. E. Villegas, N. Ramírez, M. I. Martínez, ... J. El'Gazi, Ya No es Posible el Silencio (pág. 262). Bogota: Centro de Competencia en Comunicación para América Latina Friedrich Ebert Stiftung.

\section{Notas}

[1] [2] [3][4]. Audiovisuales Comunitarios (AC); Documentales Sociales Participativos (DSP); Documentales Etnográficos (DE)

\section{Para citar este artículo}

Lopera Rueda, J. (2019). Prácticas, saberes y apropiación del medio audiovisual en los colectivos de comunicación comunitaria de la ciudad de Medellín. Revista Luciérnaga Comunicación. Vol. 11, N22. Pp. 70 - 83. Doi: https://doi.org/10.33571/ revistaluciernaga.v11 n22a2

Ojs: https://revistas.elpoli.edu.co/index.php/luc/issue/archive 